\title{
Blue Light-Induced Intracellular Movement of Phototropins: Functional Relevance or Red Herring?
}

\author{
Emmanuel Liscum ${ }^{1,2 *}$ \\ 'Division of Biological Sciences, University of Missouri, Columbia, MO, USA, ${ }^{2}$ Christopher S. Bond Life Sciences Center, \\ University of Missouri, Columbia, MO, USA
}

Keywords: phototropin, blue-light, photoreceptors, phototropism, chloroplast movement, Golgi, protein trafficking, endocytosis

The phototropin blue light (BL) photoreceptors, phot1 and phot2, mediate a number of organellar, cellular, and tissue/organ-level responses in plants including: phototropism, chloroplast accumulation and avoidance, stomatal opening, leaf expansion, and petiole/leaf orientation (Liscum et al., 2014). Not surprisingly, given the contribution of these responses to the optimization of photosynthetic efficiency and plant growth, several studies have described an intimate relationship between phot function and plant fitness (Galen et al., 2004, 2007a,b; Takemiya et al., 2005; Goh et al., 2009; Crepy and Casal, 2015). So how do phots induce such a wide

OPEN ACCESS

Edited by: Bo Liu,

University of California, Davis, USA

Reviewed by:

Xiaolei Su,

University of California, San Francisco,

USA

Tong-Seung Tseng,

Carnegie Institution for Science, USA

*Correspondence:

Emmanuel Liscum

liscume@missouri.edu

Specialty section: This article was submitted to

Plant Cell Biology,

a section of the journal

Frontiers in Plant Science

Received: 15 April 2016

Accepted: 26 May 2016

Published: 09 June 2016

Citation:

Liscum E (2016) Blue Light-Induced Intracellular Movement of

Phototropins: Functional Relevance or Red Herring? Front. Plant Sci. 7:827.

do: $10.3389 /$ fpls.2016.00827 variety of critical physiological responses? Though this remains a largely unanswered question, one apparent common early response has received recurrent attention; namely, BL-induced intracellular movement of phots (Sakamoto and Briggs, 2002; Knieb et al., 2004; Kong et al., 2006, 2007, 2013a,b; Aihara et al., 2008; Han et al., 2008; Kong and Nagatani, 2008; Sullivan et al., 2008, 2010, 2016b; Wan et al., 2008; Zienkiewicz et al., 2008; Kaiserli et al., 2009; Ding et al., 2011; Aggarwal et al., 2014; Kami et al., 2014; Preuten et al., 2015; Ishishita et al., 2016). Key observations made during these studies will be discussed here, keeping always in mind an important question: Does, or will, the study of phot movement improve our fundamental understanding of phot function, or are these events merely a red herring?

Phots are hydrophilic and contain no obvious membrane-spanning domain (Huala et al., 1997; Jarillo et al., 2001; Kagawa et al., 2001), yet it has long been known from biochemical fractionation studies that both phot 1 and phot 2 associate with the inner face of the plasma membrane in etiolated and dark-adapted plants (Gallagher et al., 1988; Palmer et al., 1993; Salomon et al., 1996; Harada et al., 2003; Knieb et al., 2004). Sakamoto and Briggs utilized Arabidopsis plants expressing a functional photl tagged with GFP to demonstrate that a fraction of phot1 is "released" from the plasma membrane to the cytoplasm in response to BL exposure (Sakamoto and Briggs, 2002). A subsequent study focused exclusively on etiolated seedlings and found that, with the exception of stomatal cells, all cells expressing the phot1-GFP protein exhibited BL-induced movement of the receptor from the plasma membrane to a "distinct mosaic pattern" within the cytoplasm (Wan et al., 2008). Moreover, the movement of phot1-GFP exhibits properties consistent with a firstorder photoreaction and occurs within minutes at high fluences of BL (Wan et al., 2008). Examples of BL-induced movement of phot1-GFP in etiolated Arabidopsis seedlings are shown in Figure 1. Cell fractionation studies have confirmed the BL-induced movement of both native and GFP-tagged phot1 from the plasma membrane to cytosolic locations (Sakamoto and Briggs, 2002; Knieb et al., 2004; Sullivan et al., 2008). Though the Sakamoto and Briggs study clearly represents the beginning of our collective fascination with the intracellular movement of phots, it is worth noting that the question of "function or red herring?" was first considered by these authors: “...it remains to be 
A

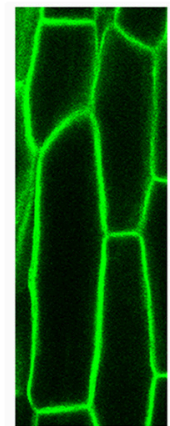

0

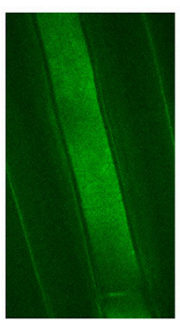

0

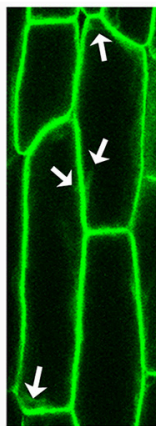

3

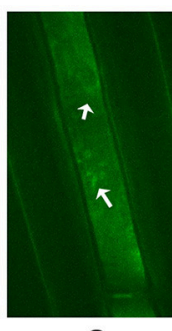

3

BL (min)
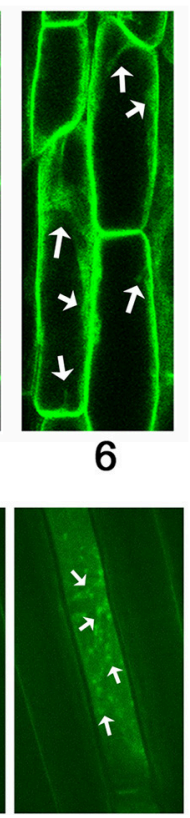

7
FIGURE 1 | Blue light-induced movement of phot1-GFP in etiolated hypocotyl cells of Arabidopsis. These images are presented as representative images of what has been observed by numerous groups many times previously. (A) Inverted confocal microscopy (Zeiss LSM 510 META) of cortical cells within the elongation zone just below the apical hook. Images are brightest point projections from midway through the cell. After identifying cells to be imaged under low intensity blue laser light (488 nm), an image was taken (time 0), and then the cells were exposed to moderate intensity blue laser light $\left(7 \mu \mathrm{mol} \mathrm{m} \mathrm{m}^{-2} \mathrm{~s}^{-1}\right)$ for the indicated times. Note, the uniform plasma membrane localization at time 0 . Arrows indicate some of the most prominent areas of cytosolic phot1-GFP moving from the plasma membrane. (B) Spinning disk confocal microscopy (Olympus IX-71 with Yokogawa spinning disk) of cortical cells within the elongation zone just below the apical hook. The focal point of the cell in the center of the image is the inner face of the plasma membrane. After identifying the cell and position to be imaged under green laser light (515 nm), an image was taken (time 0), and then the cells were exposed to moderate intensity blue laser light $(488 \mathrm{~nm})$ for the indicated times. Note the rather uniform distribution of phot1-GFP along the surface of the plasma membrane at time 0 , but punctate aggregation and formation of mosaics (arrows) after BL exposure.

determined if this light-activated shift in subcellular localization plays a signaling role, and if so, with respect to which responses" (Sakamoto and Briggs, 2002).

Not surprisingly similar experiments with plants expressing phot2-GFP soon followed. As was observed for phot1 (Sakamoto and Briggs, 2002), a vast majority of phot2-GFP protein is found associated with the plasma membrane, but a fraction of the total pool is relocalized to the cytosol in response to $\mathrm{BL}$ (Kong et al., 2006). Although some cytoplasmic phot2-GFP is also found in a "distinct mosaic pattern," like phot1 (Sakamoto and Briggs, 2002; Kong and Nagatani, 2008; Kaiserli et al., 2009), a majority is found associated with the Golgi apparatus (Kong et al., 2006, 2007, 2013a). Aggarwal and colleagues have suggested that Golgi-associated phot 2 may not be translocated from the plasma membrane per se, but rather from a small cytoplasmic fraction of phot 2 that already exists prior to BL irradiation (Aggarwal et al., 2014). Kong and colleagues have also found that BL exposure results in some phot2 (and to a lesser extent, phot1) associating with the chloroplast outer membrane (Kong et al., 2013b).

Whereas the aforementioned studies with GFP-tagged phot1 and phot 2 provided no connection between BL-induced internalization of phots and physiological function, they were sufficiently intriguing to prompt a number of additional studies aimed at defining the biochemical and cell biological parameters governing phot localization. First, it appears that the carboxylterminal portion of the protein kinase domain (PKD) of a phot is necessary for proper association with the plasma membrane, Golgi membrane, and chloroplast outer membrane (Kong et al., 2007, 2013a,b). For example, a phot1 protein lacking the carboxyl-terminal most 35 amino acids (designated phot1 $\triangle \mathrm{C} 35$ ) exhibits largely diffuse cytoplasmic localization independent of light condition (Kong et al., 2013a). A similarly truncated phot2 protein (phot $2 \Delta \mathrm{C} 42$ ) not only exhibits constitutive cytoplasmic localization, but also fails to relocalize to either the Golgi or chloroplast outer membrane in response to BL (Kong et al., 2013a,b). Second, though the PKD is necessary for membraneassociation, kinase activity is not (Kong et al., 2007; Kaiserli et al., 2009). On the other hand, PKD activity does appear essential for BL-induced relocalization of phot1 from the plasma membrane to the cytoplasm (Kaiserli et al., 2009), and for phot2 association with the Golgi (Kong et al., 2006, 2007). In the case of phot1 at least, it is not merely PKD activity but receptor autophosphorylation that is necessary for relocalization (Kaiserli et al., 2009). Results with a photoactive, but kinasedead, full-length and PKD-only phot2 proteins lead Aggarwal and colleagues to propose that it is not kinase activity, but rather the conformational changes associated with light activation of the receptor that are required for BL-induced Golgi-association of phot2 (Aggarwal et al., 2014). It is however worth noting that the latter studies were done in a Nicotiana benthamiana transient expression system (Aggarwal et al., 2014) where the kinase-dead Arabidopsis phot 2 being assayed might have been phosphorylated in trans by a native tobacco phot in response to BL exposure, as can occur with phot1 (Kaiserli et al., 2009), thus confounding interpretation of the observed results. Third, it was found that both phot 1 and phot 2 associate with clathrin at the plasma membrane (Kaiserli et al., 2009; Roberts et al., 2011; Aggarwal et al., 2014), raising the possibility that BLinduced movement of phots from the plasma membrane occurs via clathrin-mediated endocytosis. Results from pharmacological studies are consistent with this hypothesis for phot1 (Kaiserli et al., 2009), but not phot2 (Aggarwal et al., 2014). Though intuitively the latter results seem at odds with the findings that phot 2 associates with the Golgi in response to BL (Kong et al., 2006, 2007, 2013a), they are not inconsistent with the proposal that it is cytoplasmic, rather than plasma membrane-associated, phot2 that translocates to the Golgi (Aggarwal et al., 2014). Studies of phot turnover suggest that clathrin-associated phot1 and phot 2 may represent sub-pools of the receptor targeted for degradation, either as internalized protein or at the plasma membrane itself (Kaiserli et al., 2009; Sullivan et al., 2010; Roberts 
et al., 2011; Aggarwal et al., 2014). As much as these studies help refine our understanding of the BL-induced intracellular movement of phots, we are left with the same nagging question: Are these events functionally relevant or are we just chasing a red herring?

The Briggs laboratory was the first group to tackle the hard functional question. It has been known for some time that red light (RL) pre-treatment of etiolated Arabidopsis seedlings induces a phytochrome A (phyA)-dependent enhancement of subsequent BL-induced phot1-mediated phototropism (Parks et al., 1996; Janoudi et al., 1997; Stowe-Evans et al., 2001; Whippo and Hangarter, 2004; Sullivan et al., 2016a). Han and colleagues found that in seedlings pre-irradiated with RL, phot1 is no longer mobilized from the plasma membrane in response to BL irradiation (Han et al., 2008). Moreover, this retention of phot1 at plasma membrane requires phyA and exhibits the photobiological hallmarks associated with phyA-dependent enhancement of phototropism (Han et al., 2008). Based on these findings the authors proposed that increased amounts of photoactive phot 1 at the plasma membrane could positively impact lateral auxin transport to facilitate enhanced phototropism (Han et al., 2008). While not expressly stated, the results from this study also imply that cytoplasmic phot1 is not likely functional, at least with respect to phototropism.

Rather than attempting to correlate phot1 movement with a particular physiological response, Preuten and colleagues recently took a different approach by asking: What happens to phot responses if photl is irreversibly tethered to the plasma membrane (Preuten et al., 2015)? Phot1 modified with a myristoylation- or farnesylation-tag associates with the plasma membrane and does not exhibit BL-induced relocalization to the cytoplasm (Preuten et al., 2015). Expression of either of these recombinant phot 1 proteins in a phot1phot 2 double mutant results in complementation of phot1-dependent responses, including phototropism, petiole positioning, leaf flattening, and chloroplast accumulation (Preuten et al., 2015). Interestingly, RLinduced enhancement of phototropism was found to still occur in these phot1-tethered lines (Preuten et al., 2015), suggesting that the conclusions drawn by Han and colleagues about phyAdependent retention of phot 1 at the plasma membrane being potentially causative for phototropic enhancement (Han et al., 2008) may be incomplete. However, both studies implicate plasma membrane-associated phot 1 as the functionally active pool. Preuten and colleagues also found that BL-induced turnover of phot1 is normal in these transgenic lines (Preuten et al., 2015), suggesting that if clathrin-associated phot is targeted for degradation (Kaiserli et al., 2009; Sullivan et al., 2010; Aggarwal et al., 2014), that degradation occurs at the plasma membrane. At present there is no reason to expect that phot 1 would need to be internalized to be degraded as even the CRL $3^{\mathrm{NPH} 3}$ ubiquitin ligase complex that marks phot1 for BLdependent turnover resides at the plasma membrane (Roberts et al., 2011).
It would appear, based on results from Preuten et al. (2015), that the overarching question we wished to address here can be answered with a resounding: "RED HERRING." However, at least one well-characterized phot2 response defies this conclusion. Kong and colleagues have shown that the chloroplast avoidance response to high intensity BL (Jarillo et al., 2001; Kagawa et al., 2001; Sakai et al., 2001; Kong and Wada, 2016) requires association of phot 2 with the plasma membrane, Golgi membrane and chloroplast outer membrane (Kong et al., $2013 b)$. It is association with the chloroplast outer membrane in particular, which occurs only in response to $\mathrm{BL}$, that connects phot2 movement with a functional outcome (Kong et al., 2013b). As if to provide a new "siren song" to an already intrigued community, Kong and colleagues concluded: "The different intracellular localization of phototropins and/or their interactors with cellular components would be important for the precise regulation of [these] responses under fluctuating environmental light conditions" (Kong et al., 2013b).

We close by again asking: Does, or will, the study of phot movement improve our fundamental understanding of phot function, or are these events merely a red herring? With respect to phot 1 , the current evidence suggests we have wandered far down an unfulfilling path toward the "red herring," while a functional role for phot 2 relocalization appears alive. Still, the commonality of the BL-induced movement responses of phots across cell, tissue, and organ types, as well as complexity of the mechanisms regulating the responses, piques the reductionist's curiosity. Investigators will need to decide if the seduction of finding meaning in phot movement is worth the risk of continuing such studies, or if persistence is tantamount to joining the mythical hounds trained on smoked kippers who gleefully follow the scent down the rabbit hole that only leads to a potential nowhere. What about this investigator? Well, one can never meet a Cheshire Cat or Mad Hatter (Carroll, 1865) if one never risks the unknown.

\section{AUTHOR CONTRIBUTIONS}

The author confirms being the sole contributor of this work and approved it for publication.

\section{FUNDING}

Research in the Liscum laboratory is supported by the National Science Foundation (IOS-1146142).

\section{ACKNOWLEDGMENTS}

EL thanks members of his laboratory for stimulating discussions and critical reading of the manuscript. Thanks also to D. Rahm for editorial comments. Also, thanks to B. Dickinson, S. Harris, A. Smith, D. Murray, J. Gers, and N. McBrain for needed distraction. Lastly, thanks to Liscum laboratory members D. Coats Roberts and S. Askinosie for images in Figure 1. 


\section{REFERENCES}

Aggarwal, C., Banas, A. K., Kasprowicz-Maluski, A., Borghetti, C., Labuz, J., Dobrucki, J., et al. (2014). Blue-light-activated phototropin2 trafficking from the cytoplasm to Golgi/post-Golgi vesicles. J. Exp. Bot. 65, 3263-3276. doi: $10.1093 /$ jxb/eru172

Aihara, Y., Tabata, R., Suzuki, T., Shimazaki, K., and Nagatani, A. (2008). Molecular basis of the functional specificities of phototropin 1 and 2. Plant J. 56, 364-375. doi: 10.1111/j.1365-313X.2008.03605.x

Carroll, L. (1865). Alice's Adventures in Wonderland. London: Macmillian and Co.

Crepy, M. A., and Casal, J. J. (2015). Photoreceptor-mediated kin recognition in plants. New Phytol. 205, 329-338. doi: 10.1111/nph.13040

Ding, Z., Gálvan-Ampudia, C. S., Demarsy, E., Langowski, L., Kleine-Vehn, J., Fan, Y., et al. (2011). Light-mediated polarization of the PIN3 auxin transporter for the phototropic response in Arabidopsis. Nat. Cell Biol. 13, 447-452. doi: $10.1038 /$ ncb2208

Galen, C., Huddle, J., and Liscum, E. (2004). An experimental test of the adaptive evolution of phototropins: Blue-light photoreceptors controlling phototropism in Arabidopsis thaliana. Evolution 58, 515-523. doi: 10.1111/j.0014-3820.2004.tb01675.x

Galen, C., Rabenold, J. J., and Liscum, E. (2007a). Functional ecology of a blue light photoreceptor: effects of phototropin-1 on root growth enhance drought tolerance in Arabidopsis thaliana. New Phytol. 173, 91-99. doi: 10.1111/j.14698137.2006.01893.x

Galen, C., Rabenold, J. J., and Liscum, E. (2007b). Light-sensing in roots. Plant Signal. Behav. 2, 106-108. doi: 10.4161/psb.2.2.3638

Gallagher, S., Short, T. W., Ray, P. M., Pratt, L. H., and Briggs, W. R. (1988). Lightmediated changes in two proteins found associated with plasma membrane fractions from pea stem sections. Proc. Natl. Acad. Sci. U.S.A. 85, 8003-8007. doi: $10.1073 /$ pnas.85.21.8003

Goh, C. H., Jang, S., Jung, S., Kim, H. S., Kang, H. G., Park, Y. I., et al. (2009). Rice photla mutation reduces plant growth by affecting photosynthetic responses to light during early seedling growth. Plant Mol. Biol. 69, 605-619. doi: 10.1007/s11103-008-9442-1

Han, I. S., Tseng, T. S., Eisinger, W., and Briggs, W. R. (2008). Phytochrome A regulates the intracellular distribution of phototropin 1-green fluorescent protein in Arabidopsis thaliana. Plant Cell 20, 2835-2847. doi: 10.1105/tpc. 108.059915

Harada, A., Sakai, T., and Okada, K. (2003). Phot1 and phot2 mediate blue light-induced transient increases in cytosolic Ca2+ differently in Arabidopsis leaves. Proc. Natl. Acad. Sci. U.S.A. 100, 8583-8588. doi: 10.1073/pnas.13368 02100

Huala, E., Oeller, P. W., Liscum, E., Han, I. S., Larsen, E., and Briggs, W. R. (1997). Arabidopsis NPH1: a protein kinase with a putative redox-sensing domain. Science 278, 2120-2123. doi: 10.1126/science.278.5346.2120

Ishishita, K., Suetsugu, N., Hirose, Y., Higa, T., Doi, M., Wada, M., et al. (2016). Functional characterization of blue-light-induced responses and PHOTOTROPIN 1 gene in Welwitschia mirabilis. J. Plant Res. 129, 175-187. doi: 10.1007/s10265-016-0790-7

Janoudi, A. K., Konjevic, R., Whitelam, G., Gordon, W., and Poff, K. L. (1997). Both phytochrome A and phytochrome B are required for the normal expression of phototropism in Arabidopsis thaliana seedlings. Physiol. Plant. 101, 278-282. doi: 10.1111/j.1399-3054.1997.tb00997.x

Jarillo, J. A., Gabrys, H., Capel, J., Alonso, J. M., Ecker, J. R., and Cashmore, A. R. (2001). Phototropin-related NPL1 controls chloroplast relocation induced by blue light. Nature 410, 952-954. doi: 10.1038/35073622

Kagawa, T., Sakai, T., Suetsugu, N., Oikawa, K., Ishiguro, S., Kato, T., et al. (2001). Arabidopsis NPL1: a phototropin homolog controlling the chloroplast high-light avoidance response. Science 291, 2138-2141. doi: $10.1126 /$ science.291.5511.2138

Kaiserli, E., Sullivan, S., Jones, M. A., Feeney, K. A., and Christie, J. M. (2009). Domain swapping to assess the mechanistic basis of Arabidopsis phototropin 1 receptor kinase activation and endocytosis by blue light. Plant Cell 21, 3226-3244. doi: $10.1105 /$ tpc. 109.067876

Kami, C., Allenbach, L., Zourelidou, M., Ljung, K., Schutz, F., Isono, E., et al. (2014). Reduced phototropism in pks mutants may be due to altered auxinregulated gene expression or reduced lateral auxin transport. Plant J. 77, 393-403. doi: $10.1111 /$ tpj. 12395
Knieb, E., Salomon, M., and Rüdiger, W. (2004). Tissue-specific and subcellular localization of phototropin determined by immuno-blotting. Planta 218, 843-851. doi: 10.1007/s00425-003-1164-7

Kong, S. G., Kagawa, T., Wada, M., and Nagatani, A. (2013a). A C-terminal membrane association domain of phototropin 2 is necessary for chloroplast movement. Plant Cell Physiol. 54, 57-68. doi: 10.1093/pcp/pcs132

Kong, S. G., Kinoshita, T., Shimazaki, K., Mochizuki, N., Suzuki, T., and Nagatani, A. (2007). The C-terminal kinase fragment of Arabidopsis phototropin 2 triggers constitutive phototropin responses. Plant J. 51, 862-873. doi: 10.1111/j.1365-313X.2007.03187.x

Kong, S. G., and Nagatani, A. (2008). Where and how does phototropin transduce light signals in the cell? Plant Signal. Behav. 3, 275-277. doi: 10.4161/psb.3.4.5239

Kong, S. G., Suetsugu, N., Kikuchi, S., Nakai, M., Nagatani, A., and Wada, M. (2013b). Both phototropin 1 and 2 localize on the chloroplast outer membrane with distinct localization activity. Plant Cell Physiol. 54, 80-92. doi: 10.1093/pcp/pcs151

Kong, S. G., Suzuki, T., Tamura, K., Mochizuki, N., Hara-Nishimura, I., and Nagatani, A. (2006). Blue light-induced association of phototropin 2 with the Golgi apparatus. Plant J. 45, 994-1005. doi: 10.1111/j.1365-313X.2006.02667.x

Kong, S. G., and Wada, M. (2016). Molecular basis of chloroplast photorelocation movement. J. Plant Res. 129, 159-166. doi: 10.1007/s10265-016-0788-1

Liscum, E., Askinosie, S. K., Leuchtman, D. L., Morrow, J., Willenburg, K. T., and Coats, D. R. (2014). Phototropism: growing towards an understanding of plant movement. Plant Cell 26, 38-55. doi: 10.1105/tpc.113.119727

Palmer, J. M., Short, T. W., Gallagher, S., and Briggs, W. R. (1993). Blue lightinduced phosphorylation of a plasma membrane-associated protein in Zea mays L. Plant Physiol. 102, 1211-1218.

Parks, B. M., Quail, P. H., and Hangarter, R. P. (1996). Phytochrome A regulates red-light induction of phototropic enhancement in Arabidopsis. Plant Physiol. 110, 155-162. doi: 10.1104/pp.110.1.155

Preuten, T., Blackwood, L., Christie, J. M., and Fankhauser, C. (2015). Lipid anchoring of Arabidopsis phototropin 1 to assess the functional significance of receptor internalization: should I stay or should I go? New Phytol. 206, 1038-1050. doi: 10.1111/nph.13299

Roberts, D., Pedmale, U. V., Morrow, J., Sachdev, S., Lechner, E., Tang, X., et al. (2011). Modulation of phototropic responsiveness in Arabidopsis through ubiquitination of phototropin 1 by the CUL3-Ring E3 ubiquitin ligase CRL3(NPH3). Plant Cell 23, 3627-3640. doi: 10.1105/tpc.111.087999

Sakai, T., Kagawa, T., Kasahara, M., Swartz, T. E., Christie, J. M., Briggs, W. R., et al. (2001). Arabidopsis nphl and npl1: blue light receptors that mediate both phototropism and chloroplast relocation. Proc. Natl. Acad. Sci. U.S.A. 98, 6969-6974. doi: 10.1073/pnas.101137598

Sakamoto, K., and Briggs, W. R. (2002). Cellular and subcellular localization of phototropin 1. Plant Cell 14, 1723-1735. doi: 10.1105/tpc.003293

Salomon, M., Zacherl, M., and Rudiger, W. (1996). Changes in blue-lightdependent protein phosphorylation during the early development of etiolated oat seedlings. Planta 199, 336-342. doi: 10.1007/BF00195724

Stowe-Evans, E. L., Luesse, D. R., and Liscum, E. (2001). The enhancement of phototropin-induced phototropic curvature in Arabidopsis occurs via a photoreversible phytochrome A-dependent modulation of auxin responsiveness. Plant Physiol. 126, 826-834. doi: 10.1104/pp.126.2.826

Sullivan, S., Hart, J. E., Rasch, P., Walker, C. H., and Christie, J. M. (2016a). Phytochrome A mediates blue-light enhancement of secondpositive phototropism in Arabidopsis. Front. Plant Sci. 7:290. doi: $10.3389 /$ fpls.2016.00290

Sullivan, S., Kaiserli, E., Tseng, T. S., and Christie, J. M. (2010). Subcellular localization and turnover of Arabidopsis phototropin 1. Plant Signal. Behav. 5, 184-186. doi: 10.4161/psb.5.2.11082

Sullivan, S., Petersen, J., Blackwood, L., Papanatsiou, M., and Christie, J. M. (2016b). Functional characterization of Ostreococcus tauri phototropin. New Phytol. 209, 612-623. doi: 10.1111/nph.13640

Sullivan, S., Thomson, C. E., Lamont, D. J., Jones, M. A., and Christie, J. M. (2008). In vivo phosphorylation site mapping and functional characterization of Arabidopsis phototropin 1. Mol. Plant 1, 178-194. doi: 10.1093/mp/ssm017

Takemiya, A., Inoue, S., Doi, M., Kinoshita, T., and Shimazaki, K. (2005). Phototropins promote plant growth in response to blue light in low light environments. Plant Cell 17, 1120-1127. doi: 10.1105/tpc.104.030049 
Wan, Y. L., Eisinger, W., Ehrhardt, D., Kubitscheck, U., Baluska, F., and Briggs, W. (2008). The subcellular localization and blue-light-induced movement of phototropin 1-GFP in etiolated seedlings of Arabidopsis thaliana. Mol. Plant 1, 103-117. doi: 10.1093/mp/ssm011

Whippo, C. W., and Hangarter, R. P. (2004). Phytochrome modulation of bluelight-induced phototropism. Plant Cell and Environment 27, 1223-1228. doi: 10.1111/j.1365-3040.2004.01227.x

Zienkiewicz, A., Zienkiewicz, K., and Kopcewicz, J. (2008). Intracellular distribution of phototropin 1 protein in the short-day plant Ipomoea nil. Protoplasma 233, 141-147. doi: 10.1007/s00709-008-0292-0
Conflict of Interest Statement: The author declares that the research was conducted in the absence of any commercial or financial relationships that could be construed as a potential conflict of interest.

Copyright (C) 2016 Liscum. This is an open-access article distributed under the terms of the Creative Commons Attribution License (CC BY). The use, distribution or reproduction in other forums is permitted, provided the original author(s) or licensor are credited and that the original publication in this journal is cited, in accordance with accepted academic practice. No use, distribution or reproduction is permitted which does not comply with these terms. 